\title{
Human Endogenous Retroviruses-K (HML-2) Expression Is Correlated with Prognosis and Progress of Hepatocellular Carcinoma
}

\author{
Weijie Ma, ${ }^{1}$ Zhenfei Hong, ${ }^{1}$ Hailing Liu, ${ }^{2}$ Xi Chen, ${ }^{1}$ Lu Ding, ${ }^{3}$ Zhisu Liu, ${ }^{1}$ \\ Fuling Zhou, ${ }^{3}$ and Yufeng Yuan ${ }^{1}$ \\ ${ }^{1}$ Department of Hepatobiliary and Pancreatic Surgery, Zhongnan Hospital of Wuhan University, Wuhan 430071, China \\ ${ }^{2}$ Department of Clinical Hematology, Second Affiliated Hospital, Medical School, Xian Jiaotong University, Xian 710004, China \\ ${ }^{3}$ Department of Clinical Hematology, Zhongnan Hospital, Wuhan University, Wuhan, China \\ Correspondence should be addressed to Yufeng Yuan; yuanyf1971@whu.edu.cn
}

Received 4 September 2016; Revised 7 November 2016; Accepted 22 November 2016

Academic Editor: Elena Grasselli

Copyright (C) 2016 Weijie Ma et al. This is an open access article distributed under the Creative Commons Attribution License, which permits unrestricted use, distribution, and reproduction in any medium, provided the original work is properly cited.

\begin{abstract}
Background. The association between human endogenous retroviruses-K (HERV-K) (HML-2) and human disease, including a variety of cancers, has been indicated. However, the function of HERV-K (HML-2) in the progression of hepatocellular carcinoma (HCC) still remains largely unclear. Methods. We detected the expression of HERV-K (HML-2) in 84 HCC tissues and adjacent nontumor tissues by quantitative real-time PCR (qRT-PCR) and analyzed its correlation with the clinical parameters. Result. The HEVR-K level was significantly increased in HCC compared with adjacent normal tissues $(P<0.01)$ which was proved to be significantly associated with cirrhosis $(P<0.05)$, tumor differentiation $(P<0.05)$, and TNM stage $(P<0.05)$. Moreover, the high expression of HERV-K (HML-2) had a poorer overall survival than patients with lower expression by a Kaplan-Meier survival analysis $(P<0.01)$. The multivariate Cox regression analysis indicated that the level of HERV-K (HML-2) was an independent prognostic factor for the overall survival rate of HCC patients. Receiver operating characteristic (ROC) curves demonstrated the diagnostic accuracy of HERV-K (HML-2) expression in HCC (AUC $=0.729,74.7 \%$ sensitivity, and $67.8 \%$ specificity). Conclusions. Our results suggested that upregulation of HERV-K (HML-2) in HCC patients was significantly related to cancer progression and poor outcome, indicating that HERV-K (HML-2) might be a novel candidate prognostic biomarker for HCC.
\end{abstract}

\section{Introduction}

Hepatocellular carcinoma (HCC) ranks the sixth most prevalent malignant tumors and the third most common cause of cancer mortality worldwide [1]. The development of HCC is a poly-stage process involving the accumulation of genetic and epigenetic mutation of regulatory genes, which causes aberrant expression of oncogenes and tumor-suppressor gene. Massive studies have identified a large amount of molecular biomarkers, which can be used for patient assessment in multiple clinical settings, including estimating risk of disease, screening for occult primary cancers, distinguishing benign from malignant findings or one type of malignancy from another, determining prognosis and prediction for patients who have been diagnosed with cancer, monitoring status of the disease, detecting recurrence, and determining response or progression to therapy [2]. Therefore, the identification of related novel molecular biomarkers of HCC could provide new methods for the diagnosis and therapy of this malignancy.

Human endogenous retroviruses (HERVs) constitute over $8 \%$ of human genome [3]. HERVs exist as DNA remnants of infection which happened in germ lineage cell of ancient human, expand as retrotransposons within the genome and more latterly as infections agents, and have been finally inherited through generations of human [4]. These HERVs are believed to be mostly noninfectious, replication-defective, and retroviral remnants transmitted as stable mendelian genes. Since discovered in the 1980s, HERVs have been associated with autoimmune diseases and cancer 
[5, 6]. Recently, HERV mRNA, HERV proteins, and even HERV particles in human diseases, especially in cancers, have been detected. Unlike many other HERV families which have defective sequences, the HERV-K (HML-2, Human mouse mammary tumor virus like-2) family represents the most intact retroviral genes with some having open and intact reading frames for viral proteins [7]. The HERV-K family are transcriptionally active in several human cancer tissues $[8,9]$. However, the potential role of HERV-K in HCC has not been evaluated.

We aimed to evaluate the expression level of HERV-K (HML-2) in HCC patients and analyze its clinical relevance by a quantitative real-time polymerase chain reaction (qRTPCR) and to detect its potential of serving as a biomarker for predicting HCC severity and prognostic in this study.

\section{Materials and Methods}

2.1. Collection of Tissue Specimens and Clinical Features. 84 patients (78 men and 6 women, mean age $57 \pm 10$ years) who were diagnosed with HCC and underwent surgery during 2012 to 2016 were involved in this study. None of them has received preoperative chemotherapy or radiation therapy. Paired tissue specimens (tumor and adjacent normal tissues) histologically confirmed by experienced pathologists of Zhongnan Hospital of Wuhan University were collected from the patients after informing consent. Within 30 minutes after surgery, fresh tissue specimens were frozen and stored at $-80^{\circ} \mathrm{C}$ in RNAlater ${ }^{\circledR}$ RNA Stabilization Solution (Invitrogen, CA, USA) until use. Clinical and follow-up data were collected. The sixth edition of the Tumor Node Metastasis (TNM) classification system published by the International Union Against Cancer was used to define the tumor staging of HCC.

2.2. RNA Extraction and Reverse-Transcription. $100 \mathrm{mg}$ tissue specimens were used to extract RNA by the Trizol reagent (Invitrogen, CA, USA) according to the manufacturer's instructions. The extracted RNA was eluted with $40 \mu \mathrm{L}$ RNasefree water. The concentration and purity of RNA were quantified by NanoDrop ND2000 (Thermo, CA, USA). $500 \mathrm{ng}$ DNA-erased RNA was reverse transcribed in a final volume of $10 \mathrm{ul}$ following the protocol of the PrimeScript RT reagent Kit (TaKaRa, Dalian, China): $37^{\circ} \mathrm{C}$ for $15 \mathrm{~min}$ and $85^{\circ} \mathrm{C}$ for $15 \mathrm{~s}$, and then followed by storage at $4^{\circ} \mathrm{C}$.

\subsection{Quantitative Real-Time Polymerase Chain Reaction} ( $q R T-P C R)$ Analysis. For qRT-PCR, $2 \mu \mathrm{L}$ diluted reversetranscription products were mixed with $10 \mu \mathrm{L} \mathrm{SYBR}{ }^{\circledR}$ Green real-time PCR Master Mix (Toyobo, Osaka, Japan), $1 \mu \mathrm{L}$ forward primer $(10 \mu \mathrm{M}), 1 \mu \mathrm{L}$ reverse primer $(10 \mu \mathrm{M})$, and $6 \mu \mathrm{L}$ RNase-free water in a final volume of $20 \mu \mathrm{L}$. All reactions were run using the following protocol: one cycle at $95^{\circ} \mathrm{C}$ for $3 \mathrm{~min}$ and 40 cycles at $95^{\circ} \mathrm{C}$ for $15 \mathrm{~s}, 61^{\circ} \mathrm{C}$ for $3 \mathrm{~s}$, and $72^{\circ} \mathrm{C}$ for $30 \mathrm{~s}$ on CFX96 ${ }^{\mathrm{TM}}$ Real-Time System (Bio-Rad, CA, USA). At the end of the reaction, the amplification curve and melting curve were analyzed. The presence of a single peak in the melting curve analysis was applied to validate the specificity of the PCR amplification. The expression of target RNA was normalized to the expression of $18 \mathrm{~S}$ RNA. The HERV-K qPCR primers used are forward, $5^{\prime}$ - ATTGGCAACACCGTATTCTGCT $3^{\prime}$; reverse, $5^{\prime}$ - CAGTCAAAATATGGACGGATGGT - $3^{\prime}$ [10]. The primers of $18 \mathrm{~S}$ RNA were the following: forward: $5^{\prime}-$ CAGCCACCCGAGATTGAGCA - $3^{\prime}$ and reverse: $5^{\prime}$ - TAGTAGCGACGGGCGGTGTG $-3^{\prime}$. The relative expression of HERV-K was calculated by the comparative cycle threshold (Ct) method $\left(2^{-\Delta \mathrm{Ct}}\right)$.

2.4. Statistical Analysis. The SPSS version 17.0 (SPSS, Inc., Chicago, IL, USA) and GraphPad Prism 5.0 (GraphPad Software, La Jolla, CA, USA) were applied to complete statistical analyses. The Shapiro-Wilk test was applied to check the normality of the distribution. The normally distributed numeric variables were presented as mean \pm standard deviation (SD) and evaluated by Student's $t$-test, while nonnormally distributed variables were analyzed by Kruskal-Wallis Variance analysis. The categorical variables were analyzed using Chi square test. Kaplan-Meier test was used to estimate the impact of HERV-K level on the prediction of overall survival in HCC cases, and log-rank test was applied to analyze the statistical differences of different groups. The Cox proportional hazards model was assessed through multivariate analysis which contained the variables with a $P<0.05$ in univariate analysis. Receiver operating characteristic (ROC) curve and area under the ROC curve (AUC) were designed to estimate the diagnostic value. For all analyses, a $P<0.05$ (two-tail) was considered statistically significant. Statistical significance was assigned at $P<0.05(*)$ or $P<0.01(* *)$.

\section{Results}

3.1. HERV-K (HML-2) Was Upregulated in HCC Tissue Specimens. The relative expression of HERV-K (HML-2) was detected and analyzed in 84 paired clinical HCC tissues and adjacent normal liver tissues. We found that the expression level of HEVR-K was significantly increased in HCC compared with adjacent normal tissues $(P<0.01 * *$, Table 1 , Figure 1(a)).

3.2. Correlation between HEVR-K and the Pathologic Features of HCC. The association of HERV-K with clinical features of 84 HCC patients was assessed as shown in Table $1(P<$ 0.05). We found significant correlation between HERV-K and some pathologic data, such as differentiation, TNM stage, and cirrhosis (Figure 1(b)). However, either gender, age, tumor size, tumor nodes, or other clinical pathological information did not differ significantly with the highly expressed HERV-K $(P>0.05$, Table 1$)$.

3.3. High Expression of HERV-K Predicts Poor Prognosis in Patients with HCC. This cohort of 84 HCC patients was divided into low-expressed group $(<5.96, n=42)$ and highexpressed group $(\geq 5.96, n=42)$ according to the normalized median level of HERV-K (median = 5.96). The correlation between HERV-K expression and overall survival of HCC 
TABLE 1: Association of HERV-K expression with clinical parameters in HCC.

\begin{tabular}{|c|c|c|c|c|}
\hline \multirow{2}{*}{ Characteristics } & \multirow{2}{*}{$n$} & \multicolumn{3}{|c|}{ HERV-K relative expression $(-\log )$} \\
\hline & & Mean \pm SD & $t$ & $P$ \\
\hline Tissue $^{* *}$ & & & -8.224 & $<0.001$ \\
\hline HCC & 84 & $5.94 \pm 0.83$ & & \\
\hline Adjacent noncancerous liver & 84 & $6.49 \pm 0.72$ & & \\
\hline Gender & & & 0.306 & 0.240 \\
\hline Male & 78 & $5.95 \pm 0.86$ & & \\
\hline Female & 6 & $5.84 \pm 0.32$ & & \\
\hline Age & & & -0.130 & 0.320 \\
\hline$<55$ & 40 & $5.84 \pm 1.00$ & & \\
\hline$\geqq 55$ & 44 & $6.02 \pm 0.67$ & & \\
\hline Smoking & & & 1.280 & 0.204 \\
\hline Negative & 43 & $6.05 \pm 0.69$ & & \\
\hline Positive & 41 & $5.82 \pm 0.95$ & & \\
\hline Alcoholism & & & 1.662 & 0.100 \\
\hline Negative & 25 & $5.70 \pm 1.18$ & & \\
\hline Positive & 59 & $6.03 \pm 0.63$ & & \\
\hline Differentiation $^{*}$ & & & 2.601 & 0.011 \\
\hline High/moderate & 59 & $6.05 \pm 0.68$ & & \\
\hline Low & 15 & $5.43 \pm 1.23$ & & \\
\hline Missing & 10 & & & \\
\hline Size & & & 1.693 & 0.095 \\
\hline$<10 \mathrm{~cm}$ & 53 & $6.01 \pm 0.85$ & & \\
\hline$\geqq 10 \mathrm{~cm}$ & 23 & $5.66 \pm 0.76$ & & \\
\hline Missing & 8 & & & \\
\hline Tumor nodes & & & -0.728 & 0.469 \\
\hline Single & 66 & $5.91 \pm 0.88$ & & \\
\hline Multi & 10 & $6.12 \pm 0.61$ & & \\
\hline Missing & 8 & & & \\
\hline TNM stage* & & & 2.144 & 0.035 \\
\hline $\mathrm{I} \sim \mathrm{II}$ & 34 & $6.12 \pm 0.78$ & & \\
\hline III IV & 42 & $5.72 \pm 0.84$ & & \\
\hline Missing & 8 & & & \\
\hline HBV DNA (IU/mL) & & & 0.861 & 0.394 \\
\hline$<500$ & 17 & $5.90 \pm 0.68$ & & \\
\hline$\geqq 500$ & 34 & $5.68 \pm 0.95$ & & \\
\hline Missing & 33 & & & \\
\hline Cirrhosis $^{*}$ & & & -2.181 & 0.044 \\
\hline Negative & 34 & $6.11 \pm 0.65$ & & \\
\hline Positive & 14 & $5.39 \pm 1.17$ & & \\
\hline Missing & 36 & & & \\
\hline $\operatorname{AFP}(\mathrm{ng} / \mathrm{mL})$ & & & -0.137 & 0.891 \\
\hline$<200$ & 41 & $5.93 \pm 0.94$ & & \\
\hline$\geqq 200$ & 35 & $5.95 \pm 0.75$ & & \\
\hline Missing & 8 & & & \\
\hline $\operatorname{ALT}(\mathrm{u} / \mathrm{L})$ & & & 0.958 & 0.341 \\
\hline$<46$ & 48 & $6.00 \pm 0.76$ & & \\
\hline$\geqq 46$ & 32 & $5.81 \pm 0.96$ & & \\
\hline Missing & 4 & & & \\
\hline $\operatorname{AST}(\mathrm{u} / \mathrm{L})$ & & & 0.642 & 0.523 \\
\hline$<46$ & 45 & $5.97 \pm 0.66$ & & \\
\hline$\geqq 46$ & 35 & $5.86 \pm 1.04$ & & \\
\hline Missing & 4 & & & \\
\hline
\end{tabular}

Data are mean \pm SD. ${ }^{*} P<0.05$ and ${ }^{* *} P<0.01$.

A paired $t$-test was used with the analysis of the differences between HCC and adjacent noncancerous liver. Independent $t$-tests were applied to the rest of data. 


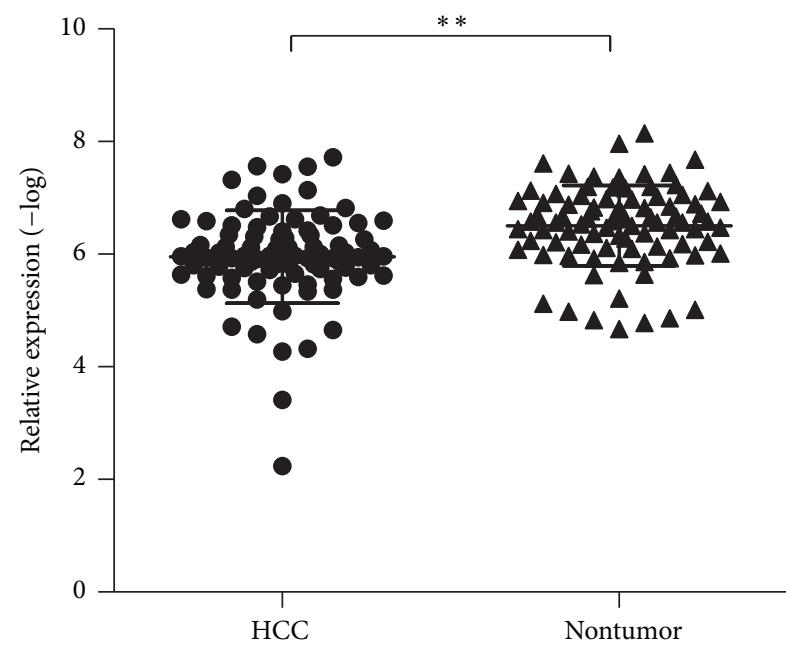

(a)

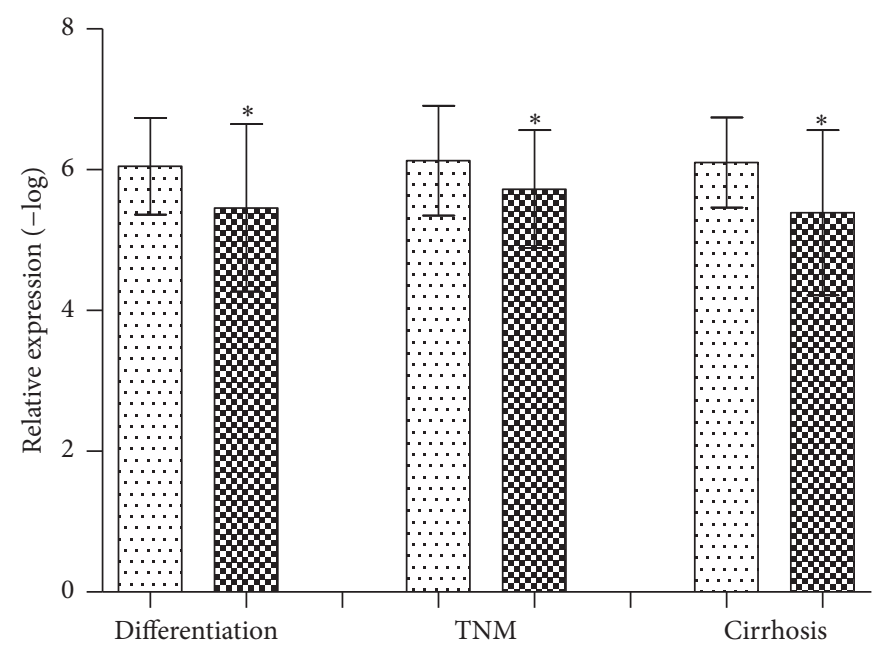

(b)

FIGURE 1: Increased HERV-K (HML-2) expression in HCC tissues and the association with clinical parameters of HCC patients. The value of relative expression was detected by the value of $-\log$ of $2^{-\Delta C t}$. (a) HERV-K (HML-2) expression level in tumor tissues was significantly higher than in nontumor tissues, $P<0.0001$. (b) Differentiation: a: high/moderate; b: low; TNM stage: a: I-II; b: III-IV; Cirrhosis: negative/positive. Results are expressed as mean $\pm \mathrm{SD}$. All data were analyzed using Student's $t$-test. ${ }^{*} P<0.05$ and ${ }^{* *} P<0.01$.

TABle 2: Prognostic factors in Cox proportional hazards model.

\begin{tabular}{|c|c|c|c|c|c|}
\hline \multirow{2}{*}{ Factors } & \multirow{2}{*}{$n$} & \multicolumn{2}{|c|}{ Univariate analysis } & \multicolumn{2}{|c|}{ Multivariate analysis } \\
\hline & & $\mathrm{HR}(95 \% \mathrm{CI})$ & $P$ & $\operatorname{HR}(95 \% \mathrm{CI})$ & $P$ \\
\hline Gender & 84 & $1.542(0.549-4.327)$ & 0.411 & & \\
\hline Age & 80 & $0.911(0.492-1.685)$ & 0.766 & & \\
\hline Smoking & 83 & $1.266(0.689-2.326)$ & 0.448 & & \\
\hline Alcoholism & 83 & $1.097(0.561-2.142)$ & 0.787 & & \\
\hline Cirrhosis & 48 & $0.813(0.362-1.826)$ & 0.616 & & \\
\hline Tumor number & 80 & $0.546(0.194-1.535)$ & 0.251 & & \\
\hline ALT & 80 & $0.978(0.528-1.812)$ & 0.944 & & \\
\hline AST & 80 & $1.013(0.551-1.861)$ & 0.967 & & \\
\hline AFP & 76 & $1.463(0.786-2.723)$ & 0.230 & & \\
\hline Differentiation & 74 & $1.866(0.903-3.853)$ & 0.092 & & \\
\hline TNM (III IV versus I II) & 76 & $4.386(2.009-9.575)$ & $<0.001$ & $3.660(1.647-8.130)$ & 0.001 \\
\hline HERV-K expression (high low versus low high) & 84 & $3.017(1.563-5.820)$ & 0.001 & $2.121(1.070-4.203)$ & 0.031 \\
\hline
\end{tabular}

$P<0.05$ was considered statistically significant; ALT: alanine aminotransferase; AST: aspartate aminotransferase; AFP: $\alpha$-fetoprotein; TNM: tumor-nodemetastasis.

patients was investigated by Kaplan-Meier analysis and logrank test. As shown in Figure 2, HCC patients with higher HERV-K expression had a tendency of shorter overall survival than those with lower HERV-K expression (low HERV$\mathrm{K}$, median: undefined; high HERV-K, median = 23; $\mathrm{HR}$ $=3.017,95 \% \mathrm{CI}=1.563-5.820$, and log-rank test: $P<$ $0.01)$. Univariate analysis indicated that tumor stage (III IV versus I II) and the expression level of HEVR-K (low versus high) were significantly associated with overall survival of HCC patients $(P<0.01$, Table 2$)$. Multivariate analysis using the Cox proportional hazards model also showed that the size of tumor, tumor stage, and HERV-K expression were independent favorable prognostic factors of $\operatorname{HCC}(P<0.05$, Table 2).

3.4. The Diagnostic Value of HERV-K in HCC. Receiver operating characteristic (ROC) curve was performed to analyze the diagnostic accuracy of HERV-K expression. The area under the ROC curve $\left(\mathrm{AUC}_{\mathrm{ROC}}\right)$ revealed that HERV-K could serve as a novel biomarker in HCC diagnosis (AUC $=0.729$, 95\% CI: $0.652-0.805,74.7 \%$ sensitivity, 67.8\% specificity, $P<0.001)$. Moreover, the expression level of HERV-K also could have high specificity in screening liver cirrhosis (100\% specificity) and high sensitivity (74.6\% sensitivity) 


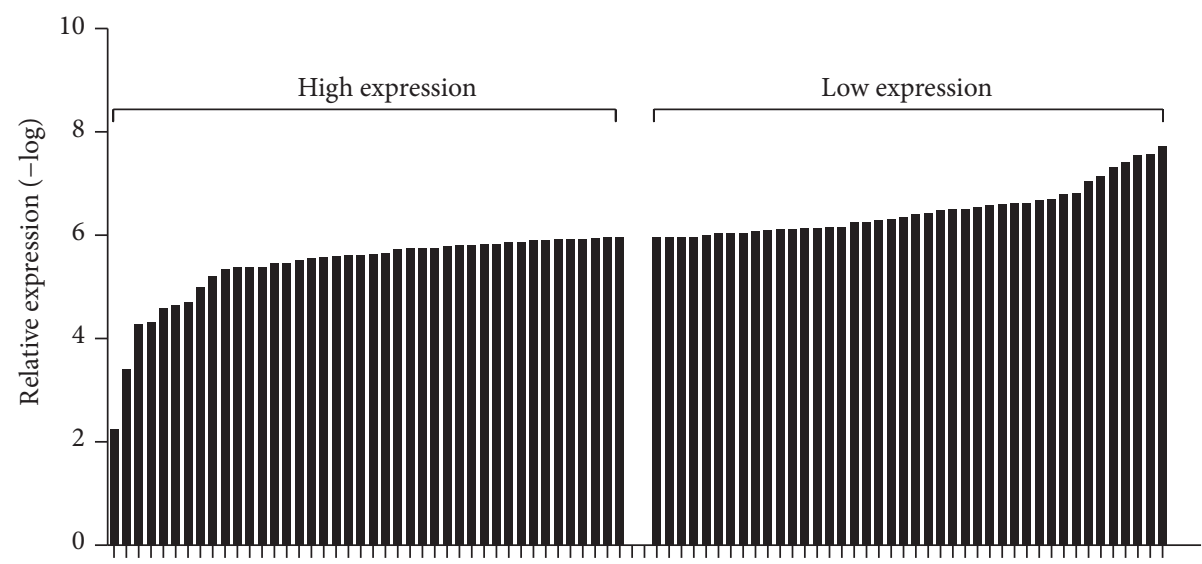

(a)

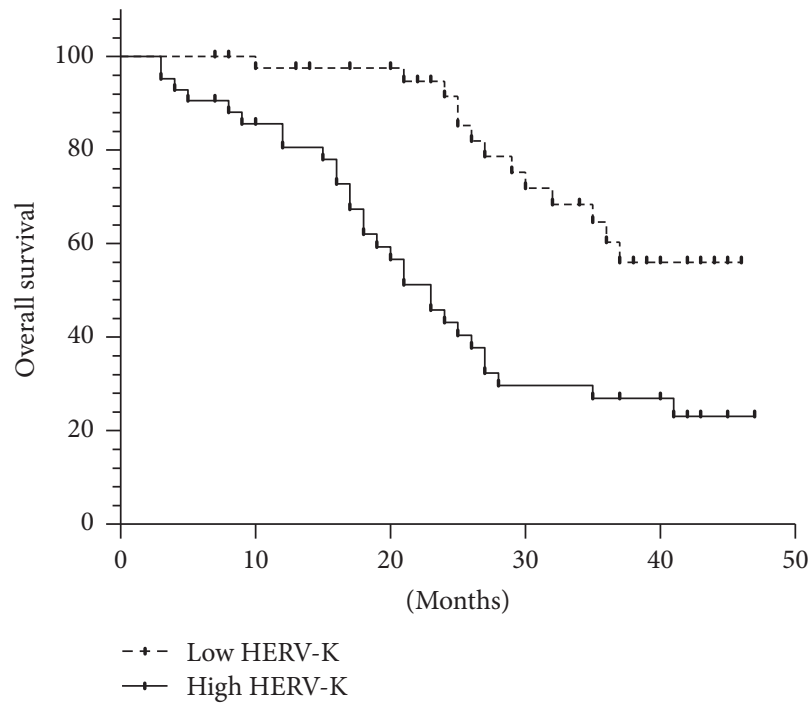

(b)

FIGURE 2: High level of HERV-K (HML-2) predicts poor survival in HCC. Patients with higher HERV-K (HML-2) expression $(n=42)$ showed reduced survival time compared with patients with lower levels of HERV-K (HML-2) expression $(n=42)$ by the Kaplan-Meier survival curve analyses (log-rank test: $P<0.01$ ).

in discriminating the grade of differentiation for HCC. Meanwhile, the ROC curve indicated that HEVR-K conferred a sensitivity of $61.8 \%$ and a specificity of $69 \%$ in division of TNM stage (Figure 3).

\section{Discussion}

HCC has the character of imperceptible pathogenesis, rapid progress, poor prognosis, and high mortality rate, which lead to tumor invasiveness, frequent intrahepatic spread and extrahepatic metastasis, and frequent recurrence after resection [11]. Owing to the lack of an effective means of clinical diagnosis at the early stage, only $30-40 \%$ of patients with HCC can be candidates for potentially curative resection by which the $5-9 \%$ of overall 5 -year survival rate can improve up to $69 \%[12,13]$. Decades of efforts have been spent on the searching of ideal biomarker for HCC [14]. Numerous biomarkers which extracted from tumor tissues or plasma have been indicated playing a vital role in predicting carcinogenesis, metastasis, and prognosis of HCC. Alpha fetal protein (AFP) was one of the conventional biomarkers for diagnosis of HCC clinically or monitoring the recurrence $[15,16]$. However, the specificity of AFP is not high enough that the detection has frequently been reported poor in the early diagnosis or the metastasis prediction of HCC [17]. On purpose of increasing the diagnostic accuracy and enhancing the effectiveness of treatment of HCC, it is quite essential to explore the novel valid tumor biomarkers and detect the mechanism of carcinogenesis.

Human endogenous retroviruses are ancestral relics of germline throughout the process of evolution. Same with all the other retroviruses, HERVs have the genomic structure: $5^{\prime}$ LTR-gag, pro, pol, and env- $3^{\prime}$ LTR. Long terminal repeats (LTRs) play roles as specifying promoter, enhancer, and polyadenylation signals that viruses insert their genetic sequences into host genomes. Without selective pressure on 


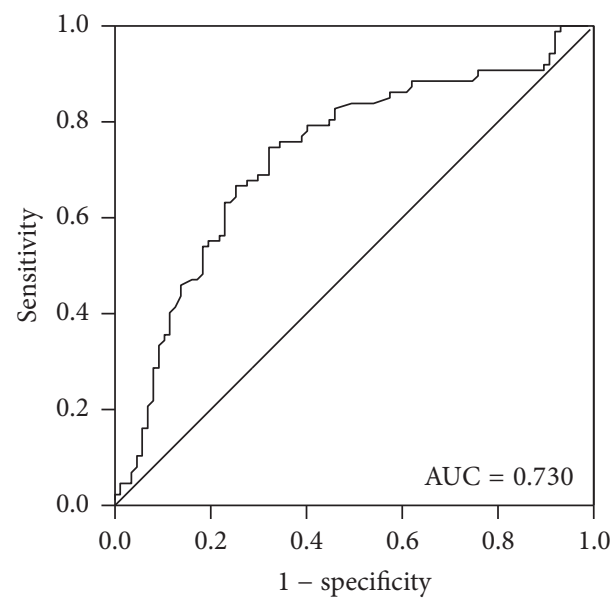

(a)

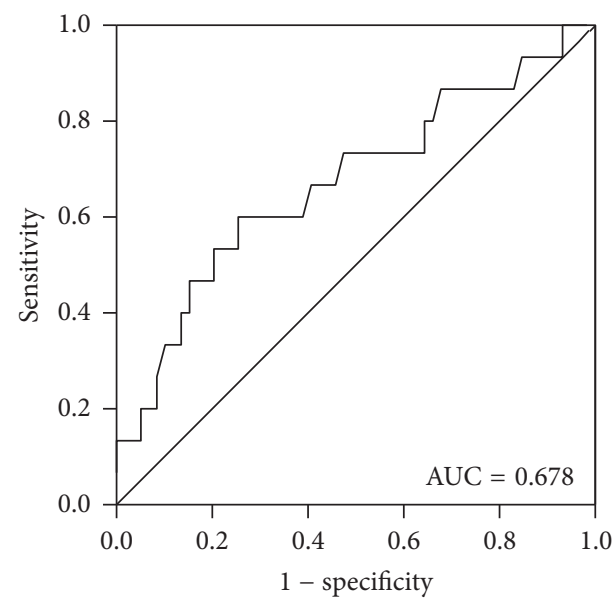

(b)

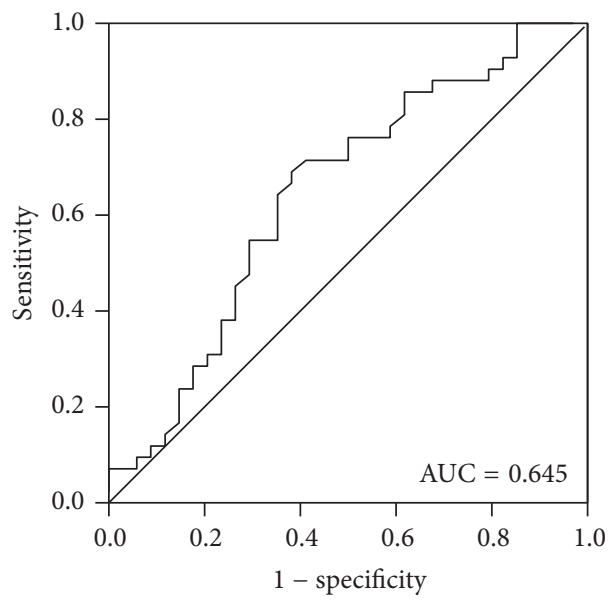

(c)

FIGURE 3: ROC curves of HERV-K (HML-2) expression. (a) HERV-K (HML-2) was considered to be a valuable parameter in the prediction of HCC (AUC $=0.730,95 \%$ CI: $65.21 \%-80.55 \%$, and $P<0.0001$ ). (b) The predicative value of HERV-K (HML-2) for differentiation of HCC resulted in AUC of 0.678 (95\% CI: $50.70 \%-83.65 \%, P=0.041)$. (c) The significant diagnostic value of HERV-K (HML-2) for TNM stage of HCC (AUC $=0.645,95 \%$ CI: $51.79 \%-77.27 \%$, and $P=0.030$ ).

the host to maintain the viral DNAs or their components in intact condition, a combination of sequence mutations, deletions, and frame-shift errors over evolutionary time has led to the loss of partial of whole genomic segments which cause homologous recombination between the two LTRs that generates solitary LTRs [18]. In most cases, HEVRs are defective owing to the multiple termination codons and deletions, while the HERV-K (HML-2) represents the most intact ERV in human genome as it maintains the open and complete reading frames.

Most members of HERV family are largely repressed in most cells under normal condition as epigenetic silencing, including HERV-K [19]. The accumulated mutations in integrated proviruses have played a role in counteracting the activity of HML-2 elements [20, 21]. Besides, DNA and histone methylation or histone deacetylation are vital mechanism for the silence of HERV-K [22]. Methyl-CpG binding domain proteins could repress the transcriptional activities of HERV-K (HML-2) [23, 24]. The action of small
RNAs, like microRNAs and Piwi-interacting RNAs (piRNAs), might be another reason for the selective expression of HEVR-K [25].

Despite these mechanisms as mentioned above, HERVs have shown their pathogenic potential both as genes/gene products and as (more or less active) retroviruses in various human diseases including autoimmune disorders, neurological, infectious diseases, and cancer [26]. In comparison with healthy tissues, the higher expression of HERV-K in malignant diseases, like melanomas [27], ovarian cancer [28], breast cancer [29], prostate cancer [30], teratocarcinoma [31], have been investigated. Smoking or ultraviolet C could be the risk factors which may have an influence on the HERV-K expression [32, 33]. Global hypomethylation and regional hypermethylation are common mechanisms of aberrant expression of gene. The loss of methylation of HERV$\mathrm{K}$ has been found in the elder which may indicate global hypomethylation or the loss of genome-wide methylation could cause the upregulation of HERV-K [34]. The enhanced 
activity of HERV-K has also been evaluated with several transcription factors. Fuchs et al. reported that the HERV-K transcription is mediated by the transcription factors Sp1 and Sp3 which are upregulated during oxidative stress and cause the gene regulation controlling multiple cellular process, like DNA damage, chromatin remodeling and proliferation, differentiation, and apoptosis of cell [35]. The long terminal repeat (LTR) of HERV-K plays a role of a TATA- and initiator element-independent promoter which has a variable transcription start site and contains four G-rich stretches playing a role of nucleosome free binding regions for Sp1 and Sp3 [35]. The increased expression of HERV-K is related to malignancies, especially hormone-associated cancers. That may demonstrate that HERV-K is susceptible to stimulation by hormones. HERV-K expression and particle release were first detected in cancers of androgen-regulated tissues such as germ cell tumors (GCT) [36]. In fact, HERV-K LTRs tend to obtain at least one putative binding site for androgen receptor, almost 800 bp upstream of the transcription start site [37]. However, more studies are needed to uncover the covert mechanism behind this phenomena.

HERV-K could be classified into two types: type 1 has $292 \mathrm{bp}$ deletion leading the expression of an abnormal $9 \mathrm{kD}$ nuclear protein named Np9 instead of a $14 \mathrm{kD}$ protein $\mathrm{Rec}$ expressed by type 2 [38]. Although either of these two proteins has interacted with various cellular proteins leading to enhanced HERV expression, Rec protein can be enhanced by androgen stimulation and independently activate the androgen receptor cascade $[39,40]$. Human small glutaminerich tetratricopeptide repeat-containing protein (hSGT) and testicular zinc finger protein (TZFP) are regulators which regulate the inactive but inducible $\mathrm{AR}$ in the cytoplasm $[39,40]$. Combined with Rec, hSGT remarkably decreased the quantity of essential ligand for activating AR and the suppression of AR-induced transactivation mediated by TZFP, resulting in augmenting HERV-K proviruses [37, 39]. Another cellular factor, promyelocytic leukemia zinc finger (PLZF) protein interacted with $\mathrm{Np} 9$ and $\mathrm{Rec}$, impairs the binding to c-myc, leading to the unbounded activity of c-myc, which in turn may lead to cancerogenic processes in germ cell tissues [41, 42].

Recently, several studies have spent efforts to uncover the molecular pathways underlying the aberrant expression of HERV-K in malignancies. It has been confirmed that the level of HERV-K is related to the status of MEK-ERK and p16INK4A-CDK4 pathways in melanoma cells [43]. The upregulated level of Np9 protein which is coded by type $1 \mathrm{HERV}-\mathrm{K}$ promoted the growth of leukemia cells in vitro and in vivo by not only activating ERK, AKT, and Notch1 pathways but also upregulating $\beta$-catenin essential for survival of leukemia stem cells [44]. In human breast cancer, the overexpressed HERV-K specifically increases Rasinduced ERK activation, which suggests that the oncogenic activity of Ras protein is propagated by activation of HERV$\mathrm{K}$ Env protein [45]. While the role which this upregulated HERV-K would play in HCC and the underlying signaling pathways are still unknown, we assume that HERV-K could be related to the transformation and tumorigenesis of HCC through Ras signaling pathways due to the detection of activated Ras/Raf/MAPK pathway in HCC [46]. Of course, this hypothesis would be fully verified in our following work.

To directly evaluate the potential roles of HERV-K in the occurrence and progress of HCC, an elaborate experiment was conducted and a rigid analysis was performed. In the present study, we confirmed that HERV-K expression is significantly upregulated in HCC tissues in comparison with adjacent normal liver tissues. In addition, intriguingly, we discovered the correlation between irregular HERV-K expression level and clinicopathological parameters in HCC. The level of HERV-K was strongly correlated with the cirrhosis, histological grade, and tumor stage in HCC patients. Moreover, we demonstrated that patients with higher level of HERV-K tended to have a poorer overall survival than patients with lower expression. Noteworthy, besides TNM stage, HERV-K expression was an independent favorable prognostic factor for patient survival through multivariate cox regression analysis. These provided an evidence that the upregulation of HERV-K in HCC might promote an increased malignant phenotype and worse prognostic phenotype. Then, ROC curves confirmed the diagnostic value of HERV-K that HEVR-K had better diagnostic value for differentiating patients with HCC from the controls.

Despite the small sample size, our study was first to provide insights into the irregular expression of HERV$\mathrm{K}$ in $\mathrm{HCC}$ and the relation between clinical features of HCC patients. We confirmed that HERV-K could be a good candidate of biomarker for diagnosis of HCC. Apparently, more additional studies are needed to be designed to explore the mechanism of HERV-K in human HCC which might shed new lights on the discovery of tumor associated antigen and the development of novel therapies for HCC further.

\section{Competing Interests}

The authors declare that they have no competing interests.

\section{Acknowledgments}

The authors would like to thank Professor Feng WangJohanning (Center for Cancer and Metabolism, SRI International, Biosciences Division, Menlo Park, Ca 94025, USA) for constructive guidance.

\section{References}

[1] A. Forner, J. M. Llovet, and J. Bruix, "Hepatocellular carcinoma," The Lancet, vol. 379, no. 9822, pp. 1245-1255, 2012.

[2] N. L. Henry and D. F. Hayes, "Cancer biomarkers," Molecular Oncology, vol. 6, no. 2, pp. 140-146, 2012.

[3] E. S. Lander, L. M. Linton, B. Birren et al., "Initial sequencing and analysis of the human genome," Nature, vol. 409, no. 6822, pp. 860-921, 2001.

[4] R. Gifford and M. Tristem, "The evolution, distribution and diversity of endogenous retroviruses," Virus Genes, vol. 26, no. 3, pp. 291-315, 2003.

[5] E. Balada, M. Vilardell-Tarrés, and J. Ordi-Ros, "Implication of human endogenous retroviruses in the development of 
autoimmune diseases," International Reviews of Immunology, vol. 29, no. 4, pp. 351-370, 2010.

[6] L. Cegolon, C. Salata, E. Weiderpass, P. Vineis, G. Palù, and G. Mastrangelo, "Human endogenous retroviruses and cancer prevention: evidence and prospects," BMC Cancer, vol. 13, article 4, 2013.

[7] J. Mayer, M. Sauter, A. Racz, D. Scherer, N. Mueller-Lantzsch, and E. Meese, "An almost-intact human endogenous retrovirus K on human chromosome 7," Nature Genetics, vol. 21, no. 3, pp. 257-258, 1999.

[8] O. Hohn, K. Hanke, and N. Bannert, "HERV-K(HML-2), the best preserved family of HERVs: endogenization, expression, and implications in health and disease," Frontiers in Oncology, vol. 3, article 246, 2013.

[9] R. F. Downey, F. J. Sullivan, F. Wang-Johanning, S. Ambs, F. J. Giles, and S. A. Glynn, "Human endogenous retrovirus K and cancer: innocent bystander or tumorigenic accomplice?" International Journal of Cancer, vol. 137, no. 6, pp. 1249-1257, 2015.

[10] F. Wang-Johanning, A. R. Frost, B. Jian, L. Epp, D. W. Lu, and G. L. Johanning, "Quantitation of HERV-K env gene expression and splicing in human breast cancer," Oncogene, vol. 22, no. 10, pp. 1528-1535, 2003.

[11] T. Yamashita and X. W. Wang, "Cancer stem cells in the development of liver cancer," The Journal of Clinical Investigation, vol. 123, no. 5, pp. 1911-1918, 2013.

[12] J. Zhou, L. Yu, X. Gao et al., "Plasma microRNA panel to diagnose hepatitis B virus-related hepatocellular carcinoma," Journal of Clinical Oncology, vol. 29, no. 36, pp. 4781-4788, 2011.

[13] A. M. Liu, T.-J. Yao, W. Wang et al., "Circulating miR-15b and miR-130b in serum as potential markers for detecting hepatocellular carcinoma: a retrospective cohort study," $B M J$ Open, vol. 2, Article ID e000825, 2012.

[14] S. Shang, A. Plymoth, S. Ge et al., "Identification of osteopontin as a novel marker for early hepatocellular carcinoma," Hepatology, vol. 55, no. 2, pp. 483-490, 2012.

[15] T. Yamashita, A. Kitao, O. Matsui et al., "Gd-EOB-DTPAenhanced magnetic resonance imaging and alpha-fetoprotein predict prognosis of early-stage hepatocellular carcinoma," Hepatology, vol. 60, no. 5, pp. 1674-1685, 2014.

[16] Q. Shen, J. Fan, X. R. Yang, Y. Tan, W. Zhao, Y. Xu et al., "Serum DKK1 as a protein biomarker for the diagnosis of hepatocellular carcinoma: a large-scale, multicentre study," The Lancet Oncology, vol. 13, no. 8, pp. 817-826, 2012.

[17] J. Tang, R. Jiang, L. Deng, X. Zhang, K. Wang, and B. Sun, "Circulation long non-coding RNAs act as biomarkers for predicting tumorigenesis and metastasis in hepatocellular carcinoma," Oncotarget, vol. 6, no. 6, pp. 4505-4515, 2015.

[18] J. Lenz, "HERV-K HML-2 diversity among humans," Proceedings of the National Academy of Sciences, vol. 113, no. 16, pp. 4240-4242, 2016.

[19] N. Bannert and R. Kurth, "Retroelements and the human genome: new perspectives on an old relation," Proceedings of the National Academy of Sciences of the United States of America, vol. 101, no. 2, pp. 14572-14579, 2004.

[20] M. Dewannieux, F. Harper, A. Richaud et al., "Identification of an infectious progenitor for the multiple-copy HERV-K human endogenous retroelements," Genome Research, vol. 16, no. 12, pp. 1548-1556, 2006.

[21] Y. N. Lee and P. D. Bieniasz, "Reconstitution of an infectious human endogenous retrovirus," PLoS Pathogens, vol. 3, no. 1, article el0, 2007.
[22] M. Dewannieux and T. Heidmann, "Endogenous retroviruses: acquisition, amplification and taming of genome invaders," Current Opinion in Virology, vol. 3, no. 6, pp. 646-656, 2013.

[23] L. Lavie, M. Kitova, E. Maldener, E. Meese, and J. Mayer, "CpG methylation directly regulates transcriptional activity of the human endogenous retrovirus family HERV-K(HML-2)," Journal of Virology, vol. 79, no. 2, pp. 876-883, 2005.

[24] S. Stengel, U. Fiebig, R. Kurth, and J. Denner, "Regulation of human endogenous retrovirus-K expression in melanomas by CpG methylation," Genes Chromosomes and Cancer, vol. 49, no. 5, pp. 401-411, 2010.

[25] K. Hanke, O. Hohn, and N. Bannert, "HERV-K(HML-2), a seemingly silent subtenant-but still waters run deep," APMIS, vol. 124, no. 1-2, pp. 67-87, 2016.

[26] M. Suntsova, A. Garazha, A. Ivanova, D. Kaminsky, A. Zhavoronkov, and A. Buzdin, "Molecular functions of human endogenous retroviruses in health and disease," Cellular and Molecular Life Sciences, vol. 72, no. 19, pp. 3653-3675, 2015.

[27] K. Büscher, U. Trefzer, M. Hofmann, W. Sterry, R. Kurth, and J. Denner, "Expression of human endogenous retrovirus $\mathrm{K}$ in melanomas and melanoma cell lines," Cancer Research, vol. 65, no. 10, pp. 4172-4180, 2005.

[28] F. Wang-Johanning, J. Liu, K. Rycaj et al., "Expression of multiple human endogenous retrovirus surface envelope proteins in ovarian cancer," International Journal of Cancer, vol. 120, no. 1, pp. 81-90, 2007.

[29] J. Zhao, K. Rycaj, S. Geng et al., "Expression of human endogenous retrovirus type $\mathrm{K}$ envelope protein is a novel candidate prognostic marker for human breast cancer," Genes and Cancer, vol. 2, no. 9, pp. 914-922, 2011.

[30] W. Goering, K. Schmitt, M. Dostert et al., "Human endogenous retrovirus HERV-K(HML-2) activity in prostate cancer is dominated by a few loci," Prostate, vol. 75, no. 16, pp. 1958-1971, 2015.

[31] N. Bhardwaj, M. Montesio, F. Roy, and J. M. Coffin, "Differential expression of HERV-K (HML-2) proviruses in cells and virions of the teratocarcinoma cell line tera-1," Viruses, vol. 7, no. 3, pp. 939-968, 2015.

[32] T. A. Wallace, R. F. Downey, C. J. Seufert et al., "Elevated HERV$\mathrm{K}$ mRNA expression in PBMC is associated with a prostate cancer diagnosis particularly in older men and smokers," Carcinogenesis, vol. 35, no. 9, pp. 2074-2083, 2014.

[33] J. Reiche, G. Pauli, and H. Ellerbrok, "Differential expression of human endogenous retrovirus $\mathrm{K}$ transcripts in primary human melanocytes and melanoma cell lines after UV irradiation," Melanoma Research, vol. 20, no. 5, pp. 435-440, 2010.

[34] P. Jintaridth and A. Mutirangura, "Distinctive patterns of age-dependent hypomethylation in interspersed repetitive sequences," Physiological Genomics, vol. 41, no. 2, pp. 194-200, 2010.

[35] N. V. Fuchs, M. Kraft, C. Tondera, K.-M. Hanschmann, J. Löwer, and R. Löwer, "Expression of the Human Endogenous Retrovirus (HERV) group HML-2/HERV-K does not depend on canonical promoter elements but is regulated by transcription factors Sp1 and Sp3," Journal of Virology, vol. 85, no. 7, pp. 34363448, 2011.

[36] K. Boller, H. Konig, M. Sauter et al., "Evidence that HERV-K is the endogenous retrovirus sequence that codes for the human teratocarcinoma-derived retrovirus HTDV," Virology, vol. 196, no. 1, pp. 349-353, 1993.

[37] M. Manghera and R. N. Douville, "Endogenous retrovirusK promoter: a landing strip for inflammatory transcription factors?" Retrovirology, vol. 10, no. 1, article 16, 2013. 
[38] V. Armbruester, M. Sauter, E. Krautkraemer et al., "A novel gene from the human endogenous retrovirus $\mathrm{K}$ expressed in transformed cells," Clinical Cancer Research, vol. 8, no. 6, pp. 1800-1807, 2002.

[39] S. Kaufmann, M. Sauter, M. Schmitt et al., "Human endogenous retrovirus protein Rec interacts with the testicular zinc-finger protein and androgen receptor," Journal of General Virology, vol. 91, no. 6, pp. 1494-1502, 2010.

[40] K. Hanke, C. Chudak, R. Kurth, and N. Bannert, "The Rec protein of HERV-K(HML-2) upregulates androgen receptor activity by binding to the human small glutamine-rich tetratricopeptide repeat protein (hSGT)," International Journal of Cancer, vol. 132, no. 3, pp. 556-567, 2013.

[41] U. M. Galli, M. Sauter, B. Lecher et al., "Human endogenous retrovirus rec interferes with germ cell development in mice and may cause carcinoma in situ, the predecessor lesion of germ cell tumors," Oncogene, vol. 24, no. 19, pp. 3223-3228, 2005.

[42] M. Denne, M. Sauter, V. Armbruester, J. D. Licht, K. Roemer, and N. Mueller-Lantzsch, "Physical and functional interactions of human endogenous retrovirus proteins $\mathrm{Np} 9$ and Rec with the promyelocytic leukemia zinc finger protein," Journal of Virology, vol. 81, no. 11, pp. 5607-5616, 2007.

[43] Z. Li, T. Sheng, X. Wan, T. Liu, H. Wu, and J. Dong, "Expression of HERV-K correlates with status of MEK-ERK and p16INK4ACDK4 pathways in melanoma cells," Cancer Investigation, vol. 28, no. 10, pp. 1031-1037, 2010.

[44] T. Chen, Z. Meng, Y. Gan et al., "The viral oncogene Np9 acts as a critical molecular switch for co-activating $\beta$-catenin, ERK, Akt and Notchl and promoting the growth of human leukemia stem/progenitor cells," Leukemia, vol. 27, no. 7, pp. 1469-1478, 2013.

[45] F. Zhou, M. Li, Y. Wei et al., "Activation of HERV-K Env protein is essential for tumorigenesis and metastasis of breast cancer cells," Oncotarget, 2016.

[46] J. M. Llovet, S. Ricci, V. Mazzaferro et al., "Sorafenib in advanced hepatocellular carcinoma," New England Journal of Medicine, vol. 359, no. 4, pp. 378-390, 2008. 


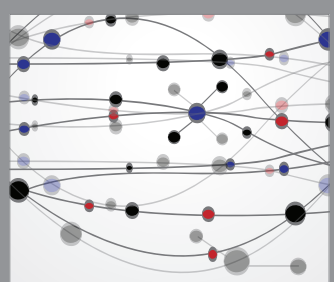

The Scientific World Journal
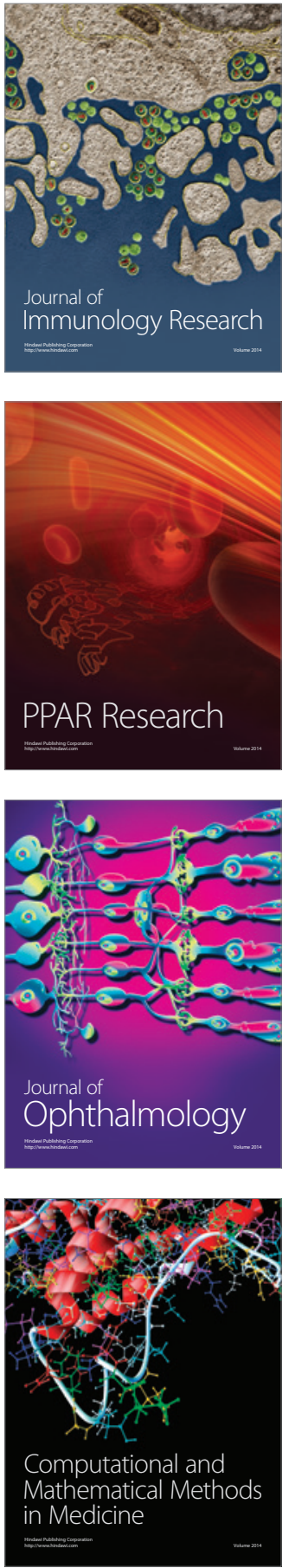

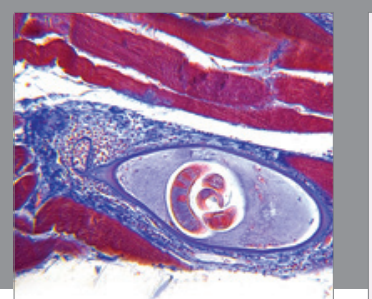

Gastroenterology Research and Practice

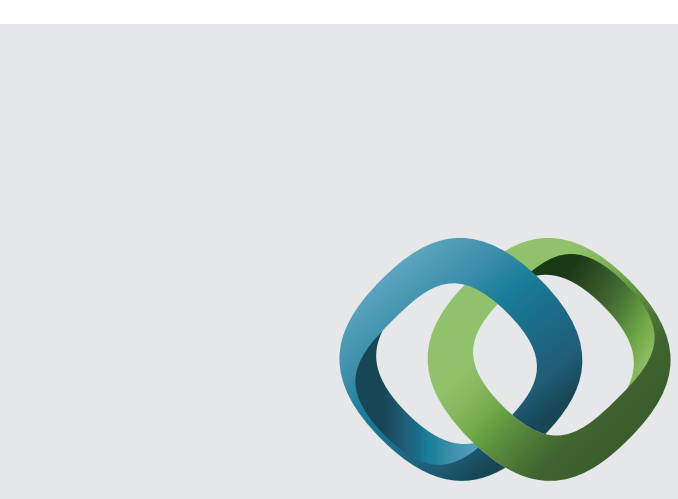

\section{Hindawi}

Submit your manuscripts at

http://www.hindawi.com
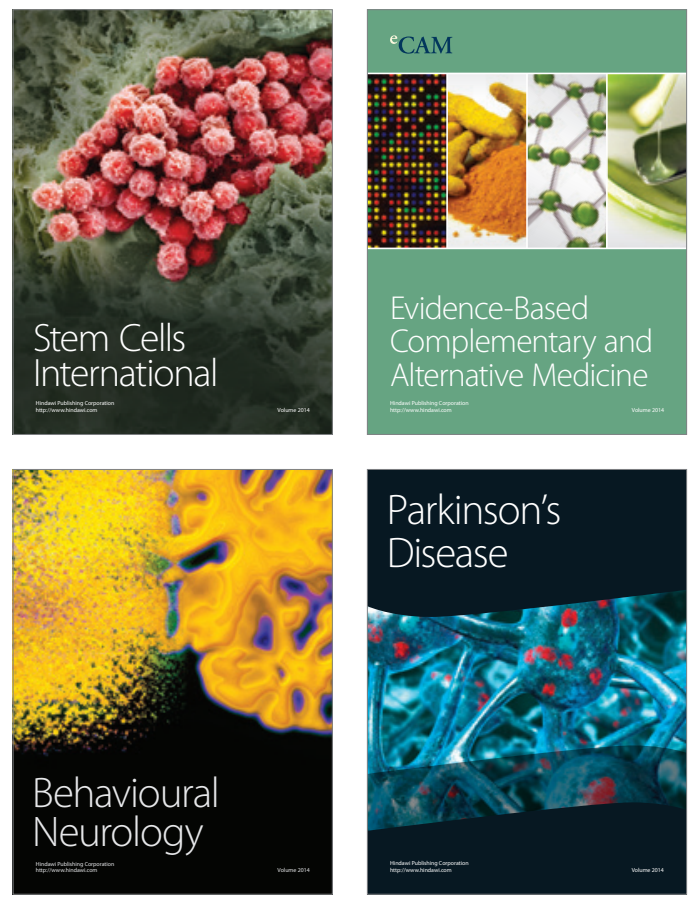
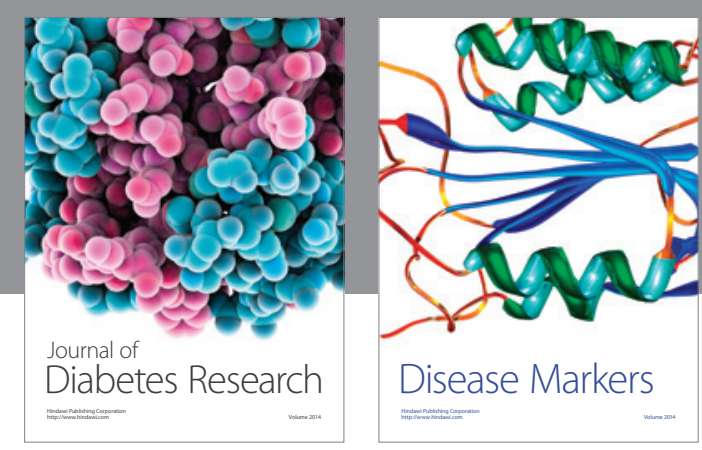

Disease Markers
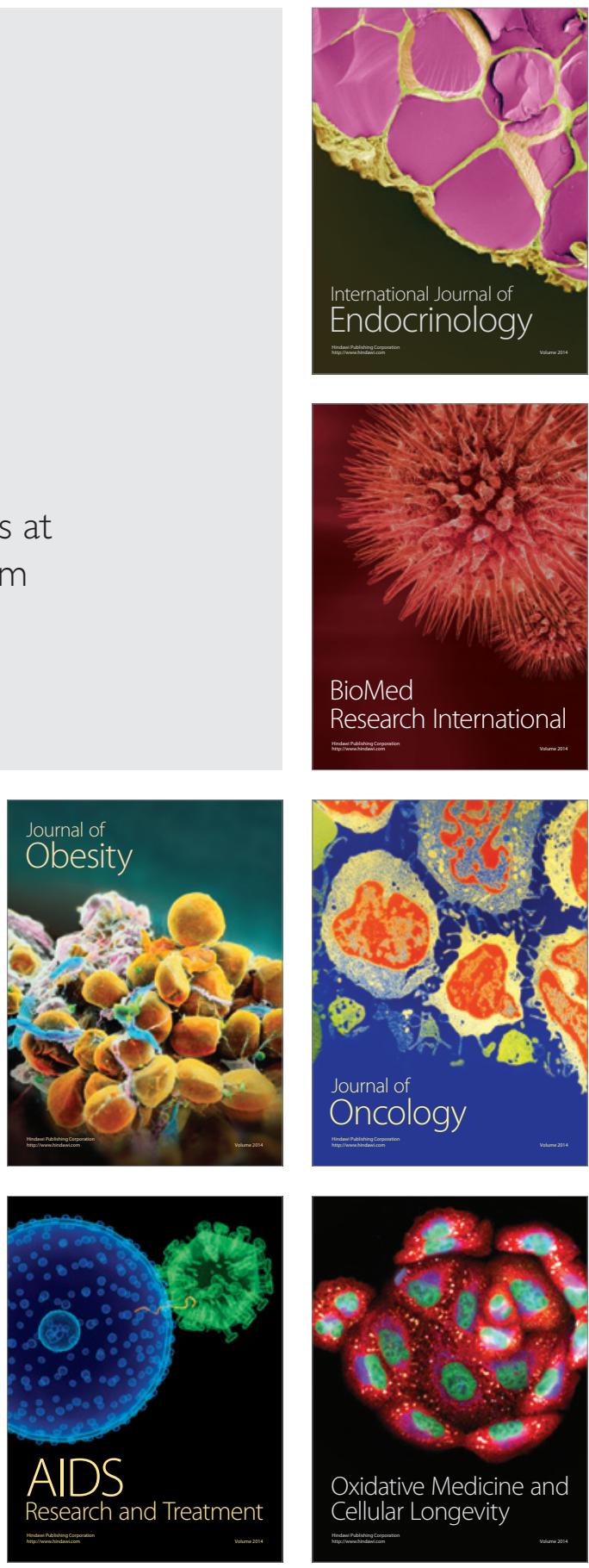